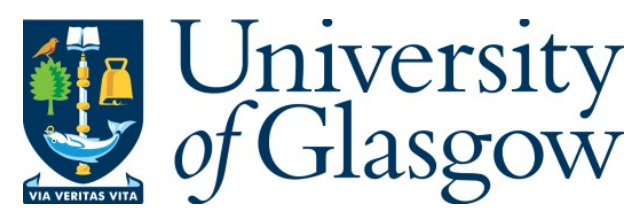

Urzagasti, D., Becerra-Alonso, D., Pérez, L. M., Mancini, H. L., and Laroze, D. (2015) Hyper-chaotic magnetisation dynamics of two interacting dipoles. Journal of Low Temperature Physics, 181(5-6), pp. 211-222.

There may be differences between this version and the published version. You are advised to consult the publisher's version if you wish to cite from it.

http://eprints.gla.ac.uk/111736/

Deposited on: 30 October 2015

Enlighten - Research publications by members of the University of Glasgow http://eprints.gla.ac.uk 


\title{
Hyper-chaotic magnetisation dynamics of two interacting dipoles
}

\author{
D. Urzagasti · D. Becerra-Alonso · L. M. \\ Pérez · H. L. Mancini · D. Laroze
}

Received: date / Accepted: date

\begin{abstract}
The present work is a numerical study of the deterministic spin dynamics of two interacting anisotropic magnetic particles in the presence of a time-dependent external magnetic field using the Landau-Lifshitz equation. Particles are coupled through the dipole-dipole interaction. The applied magnetic field is made of a constant longitudinal amplitude component and a time dependent transversal amplitude component. Dynamical states obtained are represented by their Lyapunov exponents and bifurcation diagrams. The dependence on the largest and the second largest Lyapunov exponents, as a function of the magnitude and frequency of the applied magnetic field, and the relative distance between particles, is studied. The system presents multiple transitions between regular and chaotic behaviour depending on the control parameters. In particular, the system presents consistent hyper-chaotic states.
\end{abstract}

Keywords Magnetisation Dynamics · Dipolar interaction · Hyper-Chaos

PACS $05.45 . \mathrm{Pq} \cdot 75.10 . \mathrm{Hk} \cdot 75.40 . \mathrm{Mg}$

\section{Introduction}

The combination of energy injection and dissipation maintains macroscopic systems out of the equilibrium [1]. This can generate complex dynamical states, such us noiseperiodicity, intermittency or chaos [2,3,4]. Several experiments of chaotic behaviours

D. Urzagasti

Instituto de Investigaciones Físicas, UMSA, P.O. Box 8635, La Paz, Bolivia.

D. Becerra-Alonso

Universidad Loyola Andalucía - 14004, Cordoba, Spain

L. M. Pérez · H. L. Mancini

Departamento de Física y Matemática Aplicada - Universidad de Navarra - 31080 Pamplona, Spain.

D. Laroze

SUPA School of Physics and Astronomy, University of Glasgow, Glasgow G12 8QQ, United Kingdom. Instituto de Alta de Investigación, Universidad de Tarapacá, Casilla 7D, Arica, Chile. E-mail: dlarozen@uta.cl 
in magnetic systems have been reported $[5,6,7,8,9,10]$. Typical magnetic samples are yttrium iron garnet spheres [5]. It is worth mentioning that, using the ferromagnetic resonance technique, different types of routes to chaos have been found, such as period-doubling cascades, quasi-periodic routes to chaos or intermittent routes to chaos. Therefore, a detailed characterisation of chaotic states is relevant and in order. The standard approach to study the magnetisation dynamics is based on the LandauLifshitz system, which was derived 80 years ago [11]. Using this model (or its generalisations), theoretical descriptions and phase diagrams of the chaotic regions have been given and explored[12,13,14,15,16,17, 18, 19,20,21,22]. Some of these models show new possible roots and ranges of physical parameters in chaotic domains that could motivate new experiments in this area. In addition, a deep exploration of the parameter space may help with chaos control $[23,24]$ in experimental setups.

On the other hand, applications on two particle systems under constant magnetic fields can be found in Refs. [25,26,27,28]. For conservative systems, when the interaction between the particles is based on energy exchange, it is possible to obtain analytical expressions for the magnetisations [25]. However, if the anisotropy energy is included, the system becomes non-linear and the analytical solutions are nontractable. Furthermore, the system can exhibit chaotic states in the conservative case [28]. Two interacting dipoles in the presence of an external homogeneous magnetic field were studied in Ref. [29]. The authors found that the total magnetisation is not conserved; furthermore, for the non-dissipative case it is a fluctuating function of time with a strong dependence on the strength of the dipolar term. In the dissipative case there is a transient time before the total magnetisation reaches its constant value. However, no permanent chaotic states were found. The inherent interest of this dipolar system resides in the rich variety of dynamical states it presents. Furthermore, it can be useful to understand some features of new complex materials such as artificial spin ice where the dipolar interaction plays an important role [30,31].

The aim of this paper is to analyse the influence of a time dependent external magnetic field on a system of two interacting anisotropic magnetic particles. The particles have dipole-dipole interaction. In particular, we study a periodic driving in the direction perpendicular to the main anisotropy direction, which is called the easy axis. The paper also focuses on the effect of the relative distance between particles. The dynamical behaviour studied, calculating numerically the Lyapunov exponents and other dynamical indicators, was characterised. The paper is organised in the following way. In Sec. 2, the theoretical model is briefly described. In Sec. 3, the numerical results are provided and discussed. Finally, some conclusions are presented in Sec. 4.

\section{Theoretical Model}

Let us consider two anisotropic magnetic particles in the presence of an external magnetic field, $\mathbf{H}_{\text {ext }}$. We assume that each particle can be represented by a magnetic mono-domain of magnetisation $\mathbf{M}_{i}$ with $i=(1,2)$. This approximation is called macrospin approximation. The temporal evolution of the system can be modelled by 
the Landau-Lifshitz equation [11]

$$
\frac{d \mathbf{M}_{i}}{d t}=-|\gamma| \mathbf{M}_{i} \times \mathbf{H}_{i}-\frac{\eta|\gamma|}{M_{i S}} \mathbf{M}_{i} \times\left(\mathbf{M}_{i} \times \mathbf{H}_{i}\right),
$$

where, $\gamma$ is the gyromagnetic factor, which is associated with the electron spin and is approximately given by $|\gamma|=\left|\gamma_{e}\right| \mu_{0} \approx 2.21 \times 10^{5} \mathrm{~mA}^{-1} \mathrm{~s}^{-1}$. In the above equation, $\eta$ denotes the dimensionless phenomenological damping coefficient that is characteristic of the material and has a typical value ranging from $10^{-4}$ to $10^{-3}$ in garnets and $10^{-2}$ or larger in cobalt or permalloy [32].

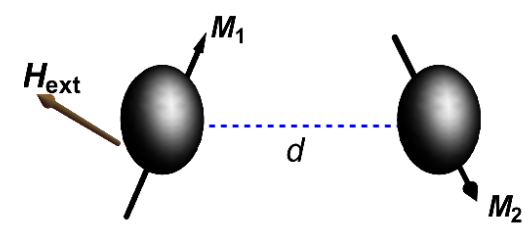

Fig. 1 (Color online) Schematic view of the two particle system. The setup of the system consists on the unit vector along the direction between the two particles is $\hat{\mathbf{r}}=\hat{\mathbf{x}}$, the anisotropy axes of particles are $\mathbf{n}_{1}=\mathbf{n}_{2}=\hat{\mathbf{z}}$, and the external field $\mathbf{H}_{\text {ext }}$, having multiple components.

We assume that the coupling between the particles is the dipole-dipole interaction, hence the effective magnetic field for each particle, $\mathbf{H}_{i}$, is given by

$$
\mathbf{H}_{i}=\mathbf{H}_{e x t}+\beta_{i}\left(\mathbf{M}_{i} \cdot \hat{\mathbf{n}}_{i}\right) \hat{\mathbf{n}}_{i}+d^{-3}\left[3\left(\mathbf{M}_{k} \cdot \hat{\mathbf{r}}\right) \hat{\mathbf{r}}-\mathbf{M}_{k}\right],
$$

being $(i, k)=1,2$ such that $i \neq k$. Here $\beta_{i}$ measures the anisotropy along the $\mathbf{n}_{i}$ axis, $d$ is the fixed distance between the two magnetic moments, and $\hat{\mathbf{r}}$ is a unit vector along the direction between the two particles. Notice that this special type of anisotropy is called uniaxial anisotropy and the constants $\beta_{i}$ can be positive or negative depending on the specific substance and sample shape [33] in use. Let us assume that the particles have the same magnitude $M_{1 S}=M_{2 S}=M_{s}$ and the same anisotropy $\beta_{1}=\beta_{2}=\beta$ and $\mathbf{n}_{1}=\mathbf{n}_{2}=\hat{\mathbf{z}}$. We apply an external magnetic field $\mathbf{H}_{\text {ext }}$ that comprises both, a constant longitudinal and a periodic transverse part with a fixed amplitude and frequency

$$
\mathbf{H}_{\text {ext }}=\mathbf{H}_{0}+\mathbf{H}_{T} \sin (\Omega t),
$$

where both $\mathbf{H}_{0}(\| \hat{\mathbf{z}})$ and $\mathbf{H}_{T}(\perp \hat{\mathbf{z}})$ are time independent. The axis $\hat{\mathbf{r}}$ is chosen perpendicular to the anisotropy axis, in particular $\hat{\mathbf{r}}=\hat{\mathbf{x}}$. Figure 1 shows a schematic view of the two particle system. Let us remark that the deterministic LL equation is only valid for very low temperatures $[11,34]$. In order to take the temperature effect into account, random magnetic field must be added [34,35]. Besides, we note that for zero damping, i.e. $\eta=0$, and without parametric forcing, i.e. $\mathbf{H}_{T}=\mathbf{0}$, Eq. (1) is conservative. Hence, the dissipation and the oscillatory injection of energy move 
the magnetic particles in and out of the equilibrium. In such a circumstance the magnetisation of the particles may exhibit complex behaviour as. e.g., quasi-periodicity, and chaos $[12,18,19,20]$. The next section provides an exhaustive characterisation of the chaotic regime including its dependence on the longitudinal field $\left|\mathbf{H}_{0}\right|$, the frequency $\Omega$ and the distance between particles $d$. This will reveal a rather complicated topology in the parameter space. The dynamical behaviour of both magnetisations is analysed using different methods.

\section{Simulations}

This Section is divided two parts. The first part briefly discusses the quantities used to characterise temporal regimes. The second part covers some numerical results and the corresponding analysis.

\subsection{Dynamical Indicators}

First, the dynamics of Eqs. (5) - (7) are characterised by evaluating the Lyapunov exponents (LEs). This method consists of quantifying the divergence between two initially close trajectories of a vector field [36,37]. In general, for an effective Ndimensional dynamical system described by a set of equations, $d X^{i} / d \tau=F^{i}(\mathbf{X}, \tau)$, the ith-Lyapunov exponent is given by

$$
\lambda_{i}=\lim _{\tau \rightarrow \infty}\left(\frac{1}{\tau} \ln \left(\frac{\left\|\delta X_{\tau}^{i}\right\|}{\left\|\delta X_{0}^{i}\right\|}\right)\right),
$$

where $\left\|\delta X_{\xi}^{i}\right\|$ is the distance between the trajectories of the ith-component of the vector field at time $\xi$. They can be ordered by decreasing amplitude: $\lambda_{1} \geq \lambda_{2} \geq$ $\ldots . \geq \lambda_{N}$. The first two exponents are the largest Lyapunov exponent (LLE) and the second largest Lyapunov exponent (SLLE). Due to the fact that the LLG equation conserves the modulus of each particle $\left|\mathbf{m}_{i}\right|$ and that the applied magnetic field is time dependent, the effective dimension of the phase space is five. From a dynamical system point of view, more than one exponent may become positive for a system of dimension five. Therefore, by exploring the dependence of the LLE on the different parameters of the system, one can identify areas in control parameter space, where the dynamics is chaotic (LLE positive), and those showing non-chaotic dynamics (LLE vanishing or negative). In addition, when both the LLE and the SLLE have positive values the system is at a hyper-chaotic regime. Nevertheless, since this is a one-frequency forced system, at least one of its Lyapunov exponents will always be zero; hence the simplest attractor is a periodic orbit. Another possibility is to have two or three Lyapunov exponents equal to zero. In these cases the system exhibits a two or three-frequency quasi-periodic behaviour. The Lyapunov exponents are presented in the form of 2-D maps as a function of the relevant parameters of the system [37, 38]. Also, a zooming technique to explore in more detail the different regimes will be used $[39,40,41]$. 
Apart from the Lyapunov spectrum analysis, there are other methods of quantifying the dynamical behaviour of a system, such as the Fourier spectrum, Poincaré sections, or correlation functions, just to mention few [10,12,17,41]. The classical technique to understand the time series of each component of $\mathbf{m}_{i}$ is to take the Fast Fourier Transform (FFT) which gives a complex discrete signal, $S(\varpi)$, in the frequency space $\varpi=\left(\varpi_{1}, \ldots, \varpi_{n}\right)$, producing a set of pairs $\left\{\varpi_{k}, S\left(\varpi_{k}\right)\right\}$. For this signal we calculate its power spectrum $|S(\varpi)|$. In general, when $|S(\varpi)|$ has a finite number of discrete peaks, the time series are regular, whereas if there is a continuum of peaks, the series may be chaotic. Let us mention that the bifurcation diagrams using Poincaré sections of the magnetisation angles, given by $\mathbf{m}_{i}=\left(\cos \phi_{i} \sin \theta_{i}, \sin \phi_{i} \sin \theta_{i}, \cos \theta_{i}\right)$, were employed in Ref. [12]. The local maximum of a specific component of $\mathbf{m}_{i}$ was used in Ref. [18]. In these diagrams, a continuum of points implies quasi-periodic or chaotic behaviour.
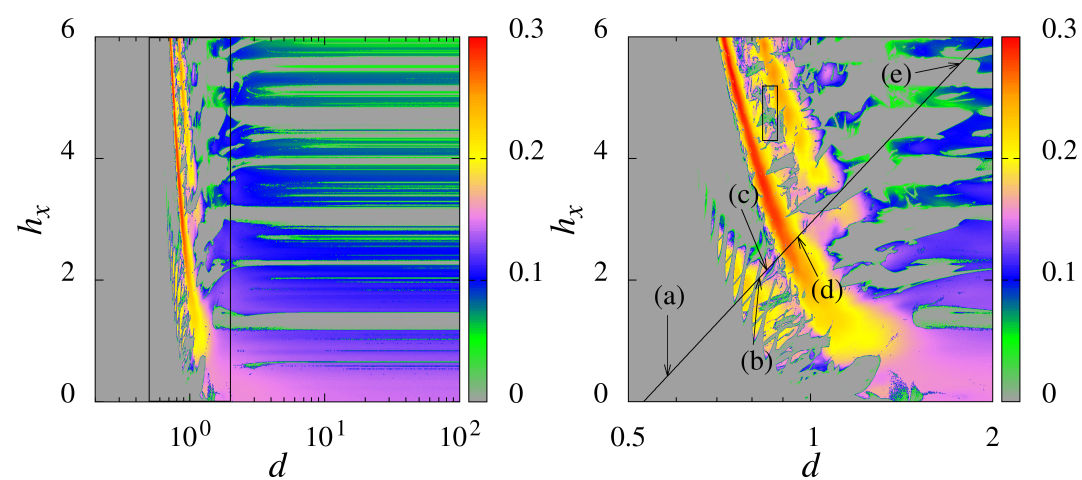

Fig. 2 (Color online) (left) Phase diagram displaying the LLE color coded as a function of the field amplitude $h_{x}$ and the distance $d$ with $\varphi=0.5, \eta=0.05, \beta=1, h_{y}=1$ and $h_{z}=0.1$. (right) Magnification of the black box in (left). The black box in (left) is shown in Fig. 5. The specific dynamics (a)-(e) along the diagonal of (left) are shown in Fig. 4.

\subsection{Numerical Results}

In order to simplify and speed-up the integration of the equations of motion, dimensionless units are used. This recasts Eq. (1) in terms of the magnetisation $\mathbf{m}_{i}=\mathbf{M}_{i} / M_{s}$ and time $\tau=t|\gamma| M_{s}$ [32]. This normalisation leads to $\left|\mathbf{m}_{i}\right|=1$. The dimensionless field is $\tilde{\mathbf{h}}_{i}=\mathbf{H}_{i} / M_{s}$ with $\tilde{h}_{x, y} \equiv h_{x, y} \sin (\varphi \tau)$, and $\tilde{h}_{z} \equiv h_{z}$ where $\varphi=\Omega /\left(|\gamma| M_{s}\right)$ is the dimensionless frequency. In order to avoid numerical artefacts [12], it is suitable to solve Eq. (1) in the Cartesian representation, namely,

$$
\begin{aligned}
\frac{d m_{x, i}}{d \tau} & =-\left(m_{y, i}+\eta m_{x, i} m_{z, i}\right) h_{z}+\left\{\left(m_{z, i}-\eta m_{x, i} m_{y, i}\right) h_{y}+\eta\left(m_{y, i}^{2}+m_{z, i}^{2}\right) h_{x}\right\} \sin (\varphi \tau) \\
& +d^{-3}\left(m_{z, k}+\eta m_{y, k} m_{x, i}\right) m_{y, i}-\left\{\beta m_{y, i}+d^{-3}\left(m_{y, k}-\eta m_{z, k} m_{x, i}\right)\right\} m_{z, i} \\
& +2 \eta d^{-3} m_{x, k} m_{y, i}^{2}-\eta\left(\beta m_{x, i}-2 d^{-3} m_{x, k}\right) m_{z, i}^{2}
\end{aligned}
$$




$$
\begin{aligned}
\frac{d m_{y, i}}{d \tau}= & \left(m_{x, i}-\eta m_{y, i} m_{z, i}\right) h_{z}-\left\{\left(m_{z, i}+\eta m_{x, i} m_{y, i}\right) h_{x}-\eta\left(m_{z, i}^{2}+m_{x, i}^{2}\right) h_{y}\right\} \sin (\varphi \tau) \\
- & \left\{\beta m_{y, i}+d^{-3} m_{y, k}\right\} \eta m_{z, i}^{2}-d^{-3}\left(m_{z, k}+2 \eta m_{x, k} m_{y, i}\right) m_{x, i}-\eta d^{-3} m_{y, k} m_{x, i}^{2} \\
+ & \left\{\beta m_{x, i}-d^{-3}\left(2 m_{x, k}-\eta m_{z, k} m_{y, i}\right)\right\} m_{z, i} \\
\frac{d m_{z, i}}{d \tau} & =\left\{\left(m_{y, i}-\eta m_{x, i} m_{z, i}\right) h_{x}-\left(m_{x, i}+\eta m_{y, i} m_{z, i}\right) h_{y}\right\} \sin (\varphi \tau) \\
& +d^{-3}\left\{\left(m_{x, i} m_{y, k}+2 m_{x, k} m_{y, i}\right)+\eta\left(m_{y, k} m_{y, i}-2 m_{x, k} m_{x, i}\right) m_{z, i}\right\} \\
& +\eta\left(m_{x, i}^{2}+m_{y, i}^{2}\right)\left\{h_{z}+\beta m_{z, i}-d^{-3} m_{z, k}\right\} .
\end{aligned}
$$

Eqs. (5) - (7) have been integrated by using a fifth order Runge-Kutta integration scheme with a variable step [49] that ensures a relative error of $10^{-7}$ on the magnetisation fields. The LEs are calculated for a time span of $\tau=80000$ after an initial transient time of $\tau=4000$ has been discarded. The Gram-Schmidt orthogonalisation process is performed after every $\delta \tau=3.91$. The error has been estimated to be $E=0.1 \%$, which is sufficiently small for the purpose of the present analysis.

In order to get a better physical insight into the problem, let us evaluate the scales introduced here. Typical experimental values of $M_{s}$ are, e.g. $M_{s}[\mathrm{Co}] \approx 1.42 \times 10^{6} \mathrm{~A} / \mathrm{m}$ for cobalt materials, and $M_{s[\mathrm{Ni}]} \approx 4.8 \times 10^{5} \mathrm{~A} / \mathrm{m}$ for nickel materials [32]. Hence, the time scale $(\tau=1)$ is in the picosecond range, $t_{s[\mathrm{Co}]}=1 /\left(|\gamma| M_{s[\mathrm{Co}]}\right) \approx 3.2 \mathrm{ps}$ and $t_{s[\mathrm{Ni}]}=1 /\left(|\gamma| M_{s[N i]}\right) \approx 6 p s$. Let us mention that in these materials the macrospin approximation (mono-domain particles) is valid for particles with sizes of $10-20 \mathrm{~nm}$, because for smaller sizes surface anisotropy effects are relevant [42] and for larger sizes non-uniform magnetic states appear, such as vortices in cobalt nanodots. In addition, the shape of the nanoparticle plays an important role in the macrospin approximation [43]. The distance between particles ranges typically from $50 \mathrm{~nm}$ to 600 nm $[31,32,33,44]$. Although the external field can be large, if it is of order of $M_{s}$, the present technology allows the generation of such magnitudes [45,46,47]. Furthermore, interesting dynamical behaviour like the magnetic analog of the inverted driven pendulum can appear [48]. Due to the large number of parameters involved in the system, $\beta=1, \eta=0.05$ and $h_{y}=1$ will be fixed throughout the paper. Parameters $\left\{h_{x}, h_{z}, \varphi, d\right\}$ will vary depending on the simulation presented.

Figure 2 shows a phase diagram displaying the LLE color coded as a function of the distance $d$ and the field amplitude $h_{x}$. It reveals that a whole region of periodicity in $d \lesssim 0.7$, within the ranges exhibited. In this region the field is not strong enough to break the consistent interaction between the dipoles. It can perturb the set as a whole, but it cannot disassociate the dipoles in independent orbits. For $d \gtrsim 3$ the dynamics for each fixed $h_{x}$ becomes very consistent, regardless of how much the distance is increased. This can therefore be considered the region where field dynamics dominates dipolar interaction. The region has interlaced chaotic and periodic dynamics as $h_{x}$ is increased. It is between these two regions, in the transition between field domination and dipole domination, i.e. $0.7 \lesssim d \lesssim 3$, where a rich variety of chaotic regions is found. Hence, a zoom on this region is performed and shown in Figure 2 (right). In this frame, the region that agrees with $h_{x} \lesssim 2.0(1-d)$ has localised patterns of rather characteristic shapes. These shapes rapidly fade away for higher fields. The value of 


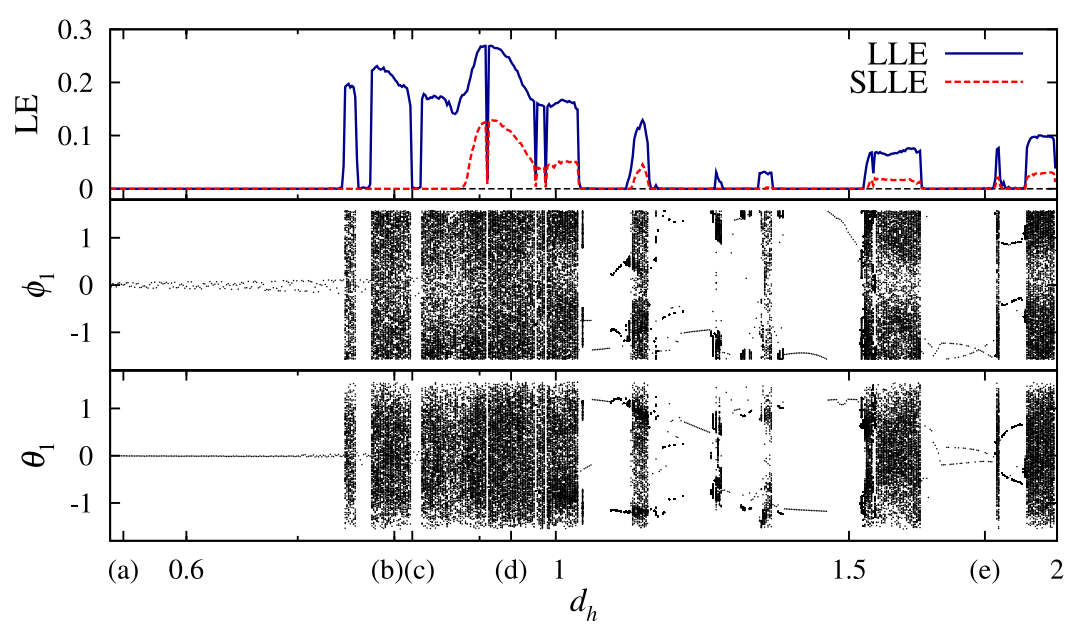

Fig. 3 (Color online) LLE and SLLE along the black line in Figure 2 (right), with the corresponding bifurcation diagrams for $\phi_{1}$ and $\theta_{1}$.

the LLE decreases and the size of the patterns decreases. The chaotic areas are not compact, but inside they contain zones with regular behaviours. A very similar fading pattern is found in [20], thus making it expectable when studying magnetic particle systems.

The LLE, the SLLE, and the maxima-based bifurcation diagram along the black line of Figure 2 (right), are presented in Figure 3. The bifurcation diagram shows no apparent change in density or distribution when the system transits from chaos into hyper-chaos. The intermittent regimes present their maxima on bifurcated values that correspond to what was obtained in Figure 2 (right): first the chaotic characteristic shapes previously mentioned, then the larger hyper-chaotic regime, and finally an iteration of chaotic, hyper-chaotic, and periodic regimes.

The particular cases specified in Figure 2 (right) are studied in more detail in Figure 4. The first column shows the trajectory of dipole 1, so it can be compared with the trajectory of dipole 2 , on the second column. The third column shows the corresponding Fourier spectrum of such trajectories. Row (a) shows the periodic state found for $d=0.550$ and $h_{x}=0.412$. It is a simple example of a synchronised periodic 3-mode state, as the Fourier plot shows. The range covered in the $\left\{m_{x}, m_{y}, m_{z}\right\}$ is very limited, indicating what would be expected for low field amplitudes and short distances: dipolar interaction dominates, allowing only a very restricted oscillation of the dipoles. For $d=0.800$ and $h_{x}=2.034$ (row (b)) the system enters unsynchronised chaos. Although the trajectories are not equal for both dipoles, a rotation around the $m_{x}$ axis is predominant in these dynamics. Both dipoles near the $m_{x, j}=\{-1,1\}$ poles, and shift to the opposite poles only to continue the spinning. The peak-spread Fourier spectrum is consistent with the positive Lyapunov exponent found for these parameters in Figure 2 (right). The system returns to periodicity when $d=0.824$ and $h_{x}=2.162$ (row (c)). The increase in both field and distance allows for a greater range of $\left\{m_{x}, m_{y}, m_{z}\right\}$ to be covered by the trajectory. However, just like in the trajec- 
(a)
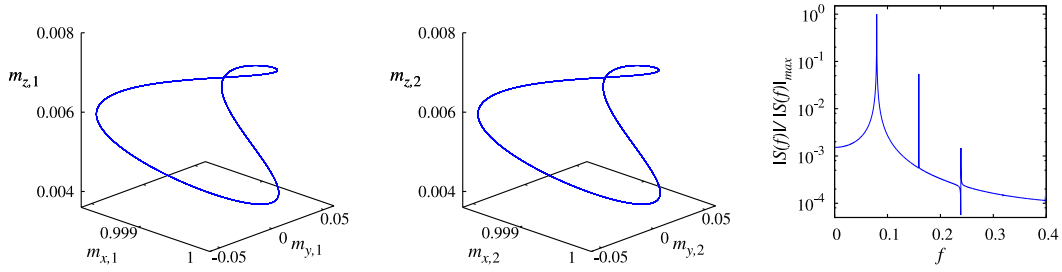

(b)
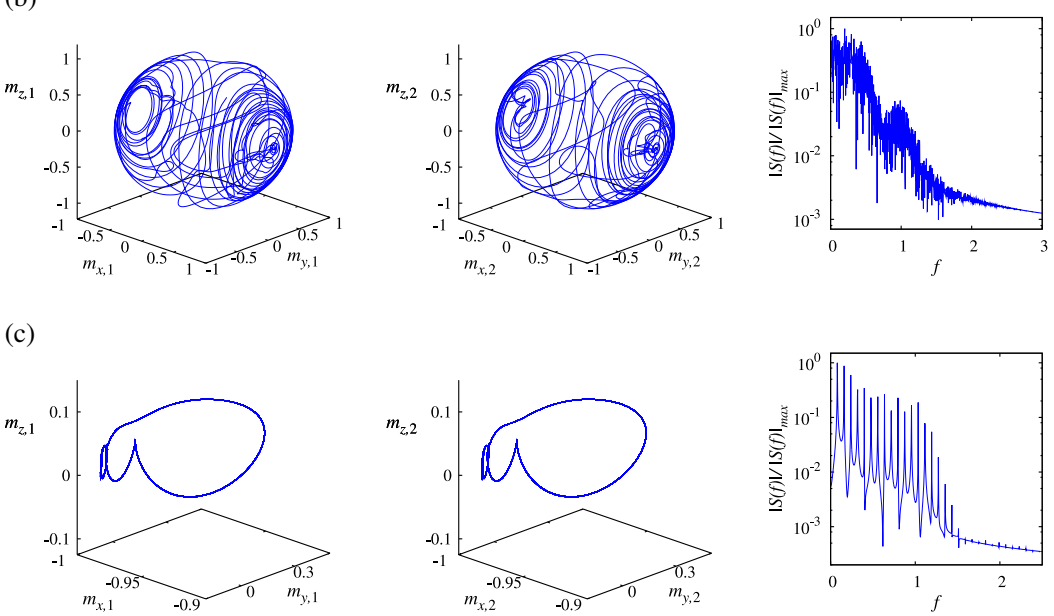

(d)
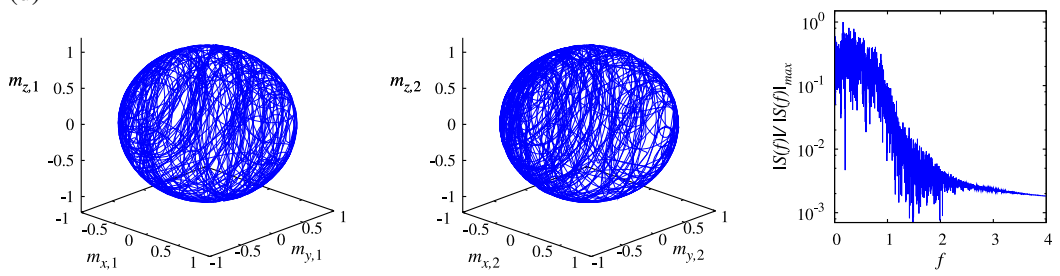

(e)
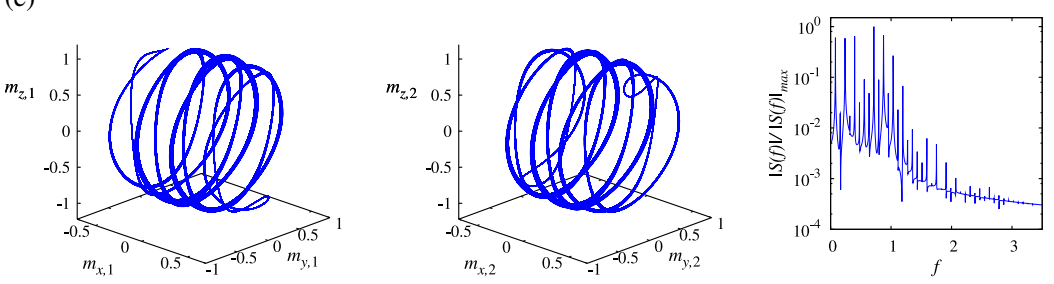

Fig. 4 Dipole trajectories (columns 1 and 2) and Fourier spectra (column 3 ) of the instances indicated in Figure 2 (right). The two first LLEs are: $\lambda_{1} \approx\left(-0.572 \times 10^{-5}, 0.203,1.6 \times 10^{-4}, 0.245,6.9 \times 10^{-4}\right)$ and $\lambda_{2} \approx\left(-5.71 \times 10^{-5},-1.3 \times 10^{-5},-3.47 \times 10^{-5}, 0.11,5.7 \times 10^{-5}\right)$, respectively. 


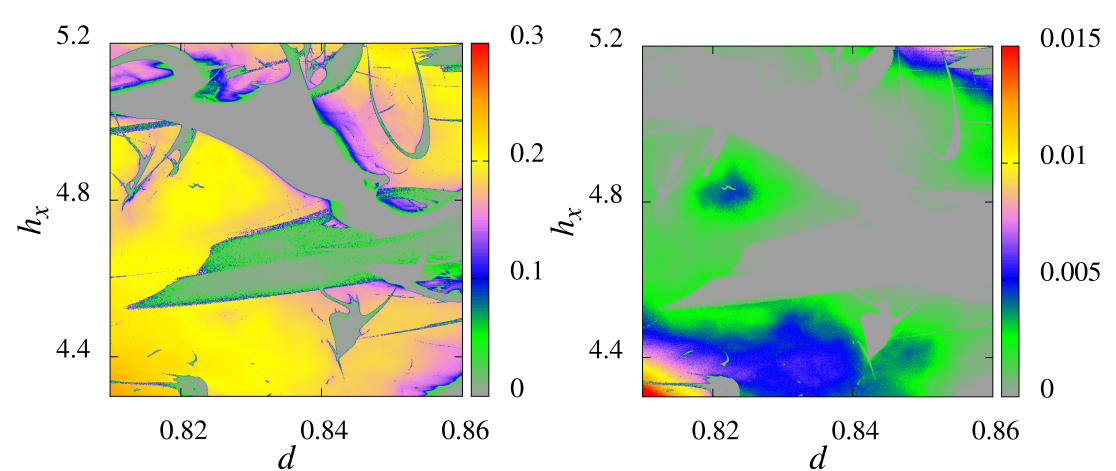

Fig. 5 (Color online) Phase diagram displaying the LLE and the SLLE color coded as a function of the field amplitude $h_{x}$ and the distance $d$. The range corresponds to the black box in Fig. 2 (right).

tories of row (a), the dynamics is confined to the neighbourhood of either $m_{x}=-1$ or $m_{x}=1$, depending on the initial conditions. A greater number of modes of oscillation is found in this synchronised periodic state, with a relatively short excursion towards the $m_{x}$ equator. Row (d) $\left(d=0.935\right.$ and $\left.h_{x}=2.709\right)$ shows hyper-chaotic trajectories for the dipoles. Although there seems to be a prevalence of rotations around the $m_{x}$ axis (as in the chaotic regime of row (b)), such instability allows for no apparent attractor. The trajectories are isotropic, and the Fourier peaks are found on a broad range of frequencies. Row (e) shows what Sparrow [3] described as noisy periodicity. The Fourier spectrum shows a number of well defined peaks in the lower frequencies, accompanied by some low-amplitude high-frequency peaks. These broaden the possible trajectories without blurring the general structure of the main periodic attractor. The result is a periodic orbit in a deterministic system that seems to be under some form of noise [3].
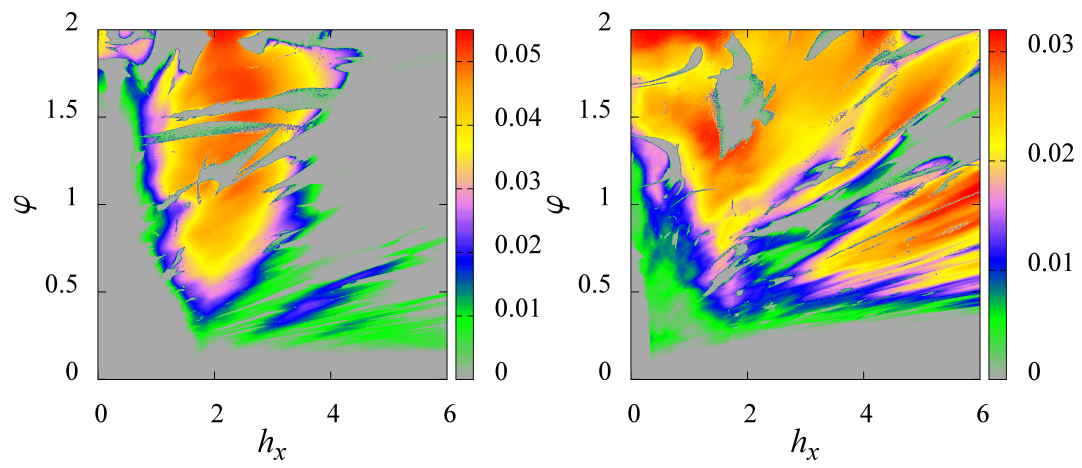

Fig. 6 (Color online) Phase diagram displaying the SLLE color coded as a function of the driven frequency $\varphi$ and the field amplitude $h_{x}$ at $h_{z}=0.1$ (left) and $h_{z}=1.0$ (right). The fixed parameters are: $d=1, \beta=1$, $h_{y}=1$ and $\eta=0.05$.

Figure 5 shows a phase diagram displaying the LLE (left panel) and the SLLE (right panel) color coded as a function of the distance $d$ and the field amplitude $h_{x}$ 
for the black box in Fig. 2 (right). We can observe that a band of periodicity with granulated chaotic regimes is found and the hyper-chaotic domains are in reduced areas of the parameter space. Hyper-chaos is, as expected, a subset of the regions with chaos. Most of the periodic-chaotic granular regions have vanished in the case of hyper-chaos, thus implying that scattered hyper-chaotic regimes in the midst of periodicity is rare, yet not inexistent. The SLLE is on average around one order of magnitude below the LLE, thus requiring a more solid refinement in the measure of the Lyapunov spectrum.

Let us explore the dependence of driven frequency in the dynamical behaviour. Figure 6 shows two phase diagrams displaying the SLLE color coded as a function of the frequency $\varphi$ and the field amplitude $h_{x}$ for two different values of the homogeneous external field $h_{z}$. The left frame shows how periodicity is mainly found in $\left\{\varphi \lesssim h_{x}-2\right\}$, in $\{\varphi \lesssim 0.2\}$, and in the rectangle defined by $\left\{\varphi \gtrsim 0.75, h_{x} \gtrsim 4\right\}$ and the edges of the frame. The rest of the frame is dominated by hyper-chaotic regimes. The larger chaotic region has a shape similar to an Arnold's tongue [50] in parametric instabilities. The right frame of Fig. 6 shows a higher value of the homogeneous field, $h_{z}=1.0$. This increment in the $h_{z}$ expands the regions with hyper-chaos. Thus, it becomes apparent that no $h_{x}$ field is needed in order to find (hyper-)chaos, when $\varphi \gtrsim 0.75$. In other words, a high enough frequency and azimuthal field suffice for unstable dynamics to occur. Periodicity dominates the regions $\left\{\varphi \lesssim 0.75\left(2.5 h_{x}-1\right), \varphi>0, h_{x}>0\right\}$ and $\left\{\varphi \lesssim 0.05 h_{x}, \varphi>0, h_{x}<6\right\}$. Chaos and hyper-chaos are found in most of the remaining region represented, with scattered islands of periodicity. The chaos-periodicity boundaries of these islands also gave granulated hyper-chaotic regimes.

\section{Final Remaks}

The magnetisation dynamics of two anisotropic magnetic particles interacting via dipolar interaction in the presence of a periodic transverse and a constant longitudinal external magnetic field has been studied using the Landau-Lifshitz equation. We have determined the regions of parameters that lead either to chaotic or to regular regimes using the Lyapunov exponent method. Extensive numerical calculations have been performed by varying two parameters simultaneously. The second largest Lyapunov exponent was computed to identify hyper chaos. This leads to phase diagrams of the second largest Lyapunov exponent, and thus of the hyper-chaotic regimes, as a function of these parameters. Generally, one finds rather intricately intermingled chaotic and regular regions separated by diffuse boundaries. The regular regions consist of either periodic or quasi-periodic solutions.

A descriptive analysis has been provided, justifying when dipole interaction dominated the field and vice-versa. Although complex and rich, the patterns presented in the parameter space have sometimes resembled structures already known from the literature [37]. An account of such coincidences has been included whenever possible. The present characterisation of the parameter space determines when the chaos is absent, and therefore it can be used as a tool to control chaos in posible experimental settings. 
Finally, an assortment of representative phase-space trajectories has been presented in this work, accounting for different kinds of periodicity, as well as chaos and hyper-chaos. The corresponding Fourier plots provide additional support to the Lyapunov spectra obtained for those particular states.

Cases of dipole synchronisation and anti-synchronisation were found in periodic, chaotic and even the hyper-chaotic regimes. Future work will present a complete study of the synchronisation phenomena.

Acknowledgements We thank R. L. Stamps (University of Glasgow, UK) for invaluable discussions. DU acknowledges the PhD fellowship from the Performance Agreement Project UTA/Mineduc (Universidad de Tarapacá). DBA was supported in part by the Spanish Inter-Ministerial Commission of Science and Technology under Project TIN2014-54583-C2-1-R, the European Regional Development fund, and the "Junta de Andalucía" (Spain), under Project P2011-TIC-7508. LMP and HLM acknowledge partial financial support from the Spanish Ministry of Science and Technology under Contract No. FIS2011-24642 and FIS2014-54101-P. DL acknowledges partial financial support from FONDECYT 1120764, Basal Program Center for Development of Nanoscience and Nanotechnology (CEDENNA) FB0807, UTA-Project 8750-12 and Engineering and Physical Sciences Research Council Grant No. EP/L002922/1.

\section{References}

1. M. C. Cross, and P.C. Hohenberg, Rev Mod. Phys. 65, 851 (1993).

2. E. N. Lorenz, J. Atmos. Sci. 20, 130 (1963).

3. C. Sparrow, The Lorenz Equations: Bifurcations, chaos and strange attractors (Springer, New York, 1982).

4. E. Ott, Chaos in dynamical systems. (Cambridge University Press, Cambridge, 2002).

5. G. Gibson, and C. Jeffries, Phys. Rev. A 29, 811 (1984).

6. F. M. de Aguiar, A. Azevedo, and S. M. Rezende, Phys. Rev. B 399448 (1989).

7. J. Becker, F. Rodelsperger, Th. Weyrauch, H. Benner, W. Just, and A. Cenys, Phys. Rev. E 59, 1622 (1999).

8. J. Cai, Y. Kato, A. Ogawa, Y. Harada, M. Chiba, and T. Hirata, J. Phys. Soc. Jap. 71, 3087 (2002).

9. M. G. Cottam (editor), Linear and Nonlinear Spin Waves in Magnetic Films and Superlattices (World Scientific, Singapore, 1992)

10. P. E. Wigen (editor), Nonlinear Phenomena and Chaos in Magnetic Materials, (World Scientific, Singapore, 1994).

11. L. Landau, Collected Papers of Landau, (Pergamon, New York, 1965).

12. L. F. Alvarez, O. Pla, and O. Chubykalo, Phys. Rev. B 61, 11613 (2000).

13. Z. Li, Y. C. Li, and S. Zhang, Phys. Rev. B 74, 054417 (2006); Phys. Rev. Lett. 99, 134101 (2007).

14. H. Z. Xu, X. Chen, and J. M. Liu, J. App. Phys. 104, 093919, (2008).

15. Y. Lan, and Y. C. Li, Nonlinearity 21, 2801(2008).

16. D. Laroze, and L. M. Perez, Physica B 403, 473 (2008).

17. D. V. Vagin, and P. Polyakov, J. Appl. Phys 105, 033914 (2009).

18. R. K. Smith, M. Grabowski, and R. E. Camley, J. Magn. Magn. Mater. 322, 2127 (2010).

19. J. Bragard, H. Pleiner, O. J. Suarez, P. Vargas, J. A. C. Gallas, and D. Laroze, Phys. Rev. E 84, 037202 (2011)

20. D. Laroze, J. Bragard, O. J. Suarez, H. Pleiner IEEE Trans. Mag. 47, 10, (2011).

21. D. Laroze, D. Becerra-Alonso, J. A. C. Gallas, and H. Pleiner, IEEE Trans. On Magnetics 48, 3567 (2012).

22. L. M. Pérez, J. Bragard, H. L. Mancini, J. A. C. Gallas, A. M. Cabanas, O. J. Suarez, and D. Laroze, Netw. Heterog. Media 10, 209 (2015).

23. T. Shinbrot, C. Grebogi, J. A. Yorke, and E. Ott, Nature 363, 411 (1993).

24. S. Boccaletti, C. Grebogi, Y.-C. Lai, H. Mancini, and D. Maza, Physics Reports 329,103 (2000).

25. D. Mentrup, J. Schnack, and M. Luban, Phys. A 272, 153 (1999).

26. D.V. Efremov, and R. A. Klemm, Phys. Rev. B 66, 174427 (2002).

27. D . Laroze, and P. Vargas, Phys. B 372, 332 (2006) 
28. L. M. Pérez, O. J. Suarez, D. Laroze, and H. L. Mancini, Cent. Eur. J. Phys. 111629 (2013).

29. D. Laroze, P. Vargas, C. Cortes, and G. Gutierrez, J. Magn. Magn. Mater. 320, 1440 (2008).

30. G. Möller , and R. Moessner, Phys. Rev. Lett. 96, 237202 (2006).

31. R. F. Wang, C. Nisoli, R. S. Freitas, J. Li, W. McConville, B. J. Cooley, M. S. Lund, N. Samarth, C. Leighton, V. H. Crespi, and P. Schiffer, Nature (London) 439, 303 (2006).

32. I. D. Mayergoyz, G. Bertotti, and C. Serpico, Nonlinear Magnetization Dynamics in Nanosystems, (Elsevier, Dordrecht, 2009).

33. R. C. O'Handley, Modern Magnetic Materials: Principles and Applications, (Wiley-Interscience, USA, 1999).

34. W. F. Brown, Jr., J. Appl. Phys. 30, 130s (1959).

35. J. L. García-Palacios, and F. J. Lázaro, Phys. Rev. B 58, 14937 (1998).

36. A. Wolf, J. B. Swift, H. L. Swinney, and J. A. Vastano, Phys. D 16, 285 (1985).

37. J. A. C. Gallas, Int. J. Bifur. Chaos, 20, 197 (2010); and references therein.

38. D. Laroze, and H. Pleiner, Commun. Nonlinear Sci. Numer. Simulat. 26, 167 (2015).

39. J. A. C. Gallas, Phys. Rev. Lett. 702714 (1993).

40. C. Bonatto, J. C. Garreau, J. A. C Gallas, Phys. Rev. Lett. 95, 143905 (2005).

41. D. Laroze, P. G. Siddheshwar, and H. Pleiner, Commun. Nonlinear Sci. Numer. Simulat. 18, 2436 (2013).

42. X. Batlle, and A. Labarta, J. Phys. D 35, R15 (2002).

43. P. Landeros, J. Escrig, D. Altbir, D.Laroze, J. d'Albuquerque e Castro, and P. Vargas, Phys. Rev. B, 65094435 (2005).

44. H. Brune, M. Giovannini, K. Bromann, and K. Kern, Nature (London) 394, 451 (1998)

45. Y. Khivintsev, B. Kuanr, T. J. Fal, M. Haftel, R. E. Camley, Z. Celinski, and D. L. Mills, Phys. Rev. B 81, 054436 (2010).

46. Y. Khivintsev, J. Marsh, V. Zagorodnii, I. Harward, J. Lovejoy, P. Krivosik, R. E. Camley, and Z. Celinski, Appl. Phys Lett. 98, 042505 (2011)

47. C. Cheng, and W. E. Bailey, Appl. Phys. Lett. 103, 242402 (2013).

48. M. G. Phelps, K. L. Livesey, A. M. Ferona, and R. E. Camley, EPL 109, 37007 (2015).

49. W. H. Press, S. A. Teukolsky, W. T. Vetterling, and B. P. Flannery, Numerical Recipes in FORTRAN (Cambridge University Press, UK, 1992).

50. R. Gilmore and M. Lefranc, The Topology of Chaos, Alice in Stretch and Squeeze Land (Wiley Interscience, USA, 2002) 